\title{
A female case of progressive familial intrahepatic cholestasis type 3 caused by heterozygous mutations of ABCB4 gene and her cirrhosis improved after treatment of ursodeoxycholic acid
}

\section{Fei Qiao}

the Affiliated Hospital of Nanjing University of Chinese Medicine

Weiting Lu

the Affiliated Hospital of Nanjing University of Chinese Medicine

Feng Ren

First Clinical Medical College, Nanjing University of Chinese Medicine, Nanjing, China

Haoran Yang

First Clinical Medical College, Nanjing University of Chinese Medicine, Nanjing, China

Guiling Mo

Guangzhou KingMed Center For Clinical Laboratory Co.,Ltd

\section{Shuangshuang Wang}

the Affiliated Hospital of Nanjing University of Chinese Medicine

Lina Liu ( $\square$ lina_liu@njucm.edu.cn )

the Affiliated Hospital of Nanjing University of Chinese Medicine

\section{Xiangtao Xu}

the Affiliated Hospital of Nanjing University of Chinese Medicine

\section{Case Report}

Keywords: Progressive familial intrahepatic cholestasis type 3 (PFIC 3), Hepatic pathology, ATP binding cassette subfamily B member 4 (ABCB4) gene, multidrug-resistant protein 3 (MDR3), ursodeoxycholic acid (UDCA)

Posted Date: February 23rd, 2022

DOI: https://doi.org/10.21203/rs.3.rs-1374900/v1

License: (c) (i) This work is licensed under a Creative Commons Attribution 4.0 International License. Read Full License 


\section{Abstract \\ Background}

Progressive familial intrahepatic cholestasis (PFIC) is a group of rapidly progressing autosomal recessive genetic diseases characterized by intrahepatic cholestasis. PFIC-3 is caused by mutations in the ATP binding cassette subfamily $B$ member 4 gene (ABCB4) encoding multidrug-resistant protein 3 (MDR3/ABCB4) protein which can lead to the absence of phosphatidylcholine, cholangiopathy, bile duct loss and biliary cirrhosis. Patients usually present in infancy or childhood, but cirrhosis and portal hypertension may be the first manifestation in older children or young people.

\section{Case presentation:}

A 25-year-old young woman with recurrent abnormal hepatic function was mainly characterized by increased gamma glutamyl transpeptisae (GGT) and bile acid with cryptogenic cirrhosis. After 7 months of treatment with ursodeoxycholic acid (UDCA), Her hepatic pathology suggested there were also obvious widening and venous fibrosis around the portal vein, and slight bile duct hyperplasia at the edge of the portal area. Infiltration of inflammatory cells around the portal vein and hepatocyte ABCB4/MDR3 protein was basically normal. Sequencing indicated the patient had heterozygous mutations in the ABCB4 gene: c. 2696C $>$ G and wes[hg19]7q21.12(87032513-87033422) $\times 1$. Through SWISS-MODEL Predict for protein structures, the missense mutation results in protein side chain missing a methyl group (-CH3), and the deletion mutation results in the serious damage to the structure of MDR3 protein. Through the analysis of pathogenicity prediction software, the mutations led to PFIC3. After treatment of UDCA for 29 months, her cirrhosis was improved, hepatic function was close to normal.

\section{Conclusion}

The novel heterozygous mutation is the molecular pathological cause of PFIC3 in this patient. Young patients with cryptogenic cirrhosis should all undergo work up for ABCB4. Diagnosis of PFIC3 early and continuous treatment of UDCA are the key to improve the prognosis and delay the occurrence of endstage liver disease.

\section{Research Background}

Progressive familial intrahepatic cholestasis (PFIC) is a group of rapidly progressing autosomal recessive genetic diseases which characterized by intrahepatic cholestasis[1]. PFIC can be usually divided into three types according to the laboratory findings, hepatic histology and pathogenic genes : PFIC-1 is also known as Byler's disease, which is caused by mutation in the ATP8B1 gene which encodes for familial intrahepatic cholestasis 1 (FIC1) protein[2] ; PFIC-2 was also previously known as Byler's syndrome [3], is caused by mutation in the ABCB 11 (ATP binding cassette [ABC] family $B$ ) encoding bile salt exporter 
pump (BSEP)[4]; and PFIC-3 is caused by mutation of $A B C B 4$ gene encoding multidrug resistance class 3 (MDR3) protein. Genes associated with FIC also include TJP2 (FIC4), NR1H4 (FIC5) and MYO5B (FIC6)[5].

PFIC-3 is caused by mutations in the ATP binding cassette subfamily B member 4 gene (ABCB4) encoding multidrug resistance class III (MDR3/ABCB4) protein, which is located on chromosome 7[6]. MDR3 transports phospholipids into the canalicular lumen to neutralize bile salts and prevents injury to biliary epithelia and bile canaliculi [7].The difference between three types is associated with high gamma glutamyl transpeptisae (GGT) in PFIC3 as compared to normal/low GGT level in patients with PFIC1 and 2 [8]. In PFIC3, hepatic histology shows portal fibrosis and small bile ductular proliferation [9], and most of the interlobular bile ducts can be displayed in the portal tracts, and some of them are reduced or disappeared [10]. PFIC3 usually manifests as repeated itching, cholestatic jaundice, and kaolin-like stools in late infancy to adolescent age; and gastrointestinal bleeding caused by cirrhosis and portal hypertension may be the first manifestation in older children or young people [11].

We reported a young female patient with an 8-year history of recurrent hepatic dysfunction, mainly manifested by elevated GGT and bile acid. After excluding other causes of hepatic function abnormalities and intrahepatic cholestasis, gene detection was finally diagnosed as a heterozygous mutation in the ABCB4 gene causing PFIC3. Gene sequence analysis revealed that this patient had a heterozygous mutations: One of the allelic genes had a new missense mutation, and the other had a large deletion mutation.

\section{Materials And Methods}

\section{Ethical compliance}

The study protocol was approved by the Ethics Committee of Affiliated Hospital of Nanjing University of Traditional Chinese Medicine. All the experiments were performed in accordance with relevant guidelines and regulations.

\section{Case data}

The patient was a young female from Jiangsu, China, who had repeated hepatic function abnormalities for 8 years without regular treatment. In March 2019, the patient was 25 years old and she came to Affiliated Hospital of Nanjing University of Traditional Chinese Medicine for the first time. In the previous year of 2018, this woman had symptoms such as jaundice, fatigue and itching and after taking transaminase-lowering drugs, the patients had no obvious clinical manifestation. Both her mother and her mother' sister's daughter had developed transient cholestasis and skin itching during their pregnancy, and then healed on their own.

\section{Clinical manifestation and diagnostic process}

The hepatic function of the patient was mainly characterized by increased GGT and bile acid. Both upper abdominal CT (September 5, 2018) and B-ultrasound (March 23, 2019) indicated hepatic cirrhosis, portal 
hypertension, splenomegaly and varicose veins in splenic hilar region, a small amount of ascites, chronic cholecystitis, and gallbladder stones. The patient was excluded from alcoholic, viral, drug-induced to nonalcoholic steatohepatitis. All autoimmune antibodies, including antimitochondrial, antinuclear, antineutrophil cytoplasmic, liver cytosol type 1 , smooth muscle,liver kidney microsome type 1 , soluble liver antigen, SP100 and GP210 antibodies were all negative. Ceruloplasmin, a-antitrypsin, transferrin saturation, thyroid function, coagulation function were all normal. Therefore, the etiology of posthepatitic cirrhosis remains to be investigated. In April 2019, the patient started taking ursodeoxycholic acid (UDCA) and drugs that could reduce transaminase. After 7 months of treatment, the patient was hospitalized for hepatic biopsy on November 5,2020 . The results of hepatic function tests during the patient's course of disease are shown inTable 1.

\section{Hematoxylin-eosin and Immunohistochemistry staining of hepatic tissue}

The hepatic tissue was fixed with $10 \%$ phosphate buffered formalin for one day, then embedded in paraffin, and then cut into- $4 \mu \mathrm{m}$ sections. After dewaxing, the sections were stained with hematoxylin and eosin (H\&E Staining Kit. no. ab245880; Abcam, Inc.) at room temperature for 3 min, dehydrated with different concentrations of ethanol. After being transparentized with xylene, the slices were sealed with neutral gum.

Antigen retrieval was performed by incubating the sections for 10 minutes at $97^{\circ} \mathrm{C}$ in $1 \mathrm{mM}$ EDTA buffer (pH 9.0). Subsequently, sections were incubated with the primary anti-bodies rabbit anti CD3 (1:100; cat. no. ab5690; Abcam, Inc.) or rabbit anti-CD20 (1:100, cat. no. ab64088; Abcam, Inc.) or rabbit Anti-ABCB4 (1:4000; cat. no. ab272457; Abcam, Inc.) at $4^{\circ} \mathrm{C}$ overnight and the biotinylated secondary antibody goat anti-rabbit immunoglobulin $\mathrm{G}$ conjugated to horseradish peroxidase (HRP)(1:300; cat. no. ab7090; Abcam, Inc) was incubated at room temperature for $30 \mathrm{~min}$. Use DAB Horseradish Peroxidase Color Development Kit, ( cat. no. P0203, Shanghai beyotime Biotechnology Co., Ltd.) for DBA color development. The images were collected and analyzed under a microscope.

\section{Exon group capture and sequencing}

\section{Whole exome sequencing and preparation of DNA library}

The genomic DNA sample of the patient was extracted from peripheral blood sample using QIAGEN DNA Blood Mini Kit (Catt. no. 51106, QIAGEN Co., Ltd.) following the Handbook.

Whole exome library was prepared using the XGen® Exome Research Panel which consists of 429,826 xGen Lockdown ${ }^{\circledR}$ Probes that span a $39 \mathrm{Mb}$ target region (19,396 genes), covering $51 \mathrm{Mb}$ of end-to-end tiled space. The full list of the genes is available online (http://sg.idtdna.com). The prepared library was loaded on the gene sequencer for sequencing (Catt. no. Novaseq 6000, Illumina, Inc.).

\section{Processing of sequencing data and bio-informatics analysis}


The sequencing data were processed with Burrows-Wheeler Aligner for alignment on a reference sequence of hg19 and Genome Analysis Toolkit for variant calling. Then, the Variant Call format files were analyzed using the ANNOVAR tools [12], which contains annotation databases, such as the 1000 genome database, dbSNP database (dbSNP; http://www.ncbi.nlm.nih.gov/SNP), ClinVar database (ClinVar; http://www.ncbi.nlm.nih.gov/clinvar), the Polymorphism Phenotyping v2 database (Polyphen-2; http://genetics.bwh.harvard.edu/pph2), and the Sorting Intolerant from Tolerant database (SIFT; http://sift.jcvi.org).

The average read depth of the whole exome sequencing was 100x. The variants were filtered under the condition of read depth $>20$, population frequency not higher than $2 \%$ in $1000 \mathrm{G}$ database and dbSNP database. All of the variants of certain pathogenicity found in the proband were went through carefully. Pathogenicity assessment of the variants was done according to the ACMG guidelines [13]. PCR primers were designed for the sites of suspected candidate mutations for amplification and Sanger sequencing verification, and the corresponding sites of the parents of the woman were detected.

\section{Sanger sequencing verification}

The Next Generation Sequencing (NGS) technology detects the positive site of ABCA4 gene, and further uses Sanger gene sequencing to verify the DNA samples of patients and their parents. According to the ABCA4 gene sequence NG_009073.1 on GenBank, primers were designed with Primer premier 5.0 software to amplify the 22nd exon of ABCA4 gene and its flanking sequences. The amplification primers and PCR conditions are shown in Table 2. After the PCR products were purified by agarose gel electrophoresis, they were subjected to capillary electrophoresis sequencing on a Genetic analyzer (3730XL, Applied Biosystems, Inc.). The sequencing results were analyzed using Mutation Surveyor software.

\section{Semi-quantitative PCR technology detection}

High-throughput sequencing methods suggest that there is a heterozygous deletion at chr7:8703342287032513, involving part of exon 27 of the ABCB4 gene, which was verified by QPCR. Primer premier 5.0 software was used to design primers, as shown in Table 3区the ABCA4 gene exon 27 and its flanking sequences were amplified in 5 segments $\bigotimes$ the amplification primers and PCR conditions are shown in Table 1. The PCR products were quantitatively analyzed onGeneration Sequencing Gene Analyzer (cat. no.7500, Applied Biosystems, Inc.).

\section{Predict the structure of mutant proteins by SWISS-MODEL}

The ABCB4 gene sequence (NM_000443) was obtained from NCBI Gene (https://www.ncbi.nlm.nih.gov/). The three-dimensional structure of the wild type and mutant (p.A899G, c.3487_3571del) of the MDR3 protein model was constructed separately, and generated by the automated protein homology modeling server Swiss-Model (https://swissmodel.expasy.org/interactive), and the MDR3 wild type Types and 
mutants use 6s7p.1.A as the template, and the three-dimensional protein structure map is generated using the protein three-dimensional structure visualization software PyMOL 2.5.

\section{Pathogenicity prediction of mutant proteins}

The pathogenicity of the detected variant was predicted by using multiple prediction software algorithms $\llbracket$

PROVEAN (http://provean.jcvi.org/protein_batch_submit.php?species=human);MutationTaster (http://www.mutationtaster.org/ChrPos.html); HGMD (http://www.hgmd.cf.ac.uk/ac/index.php).

By MutationTaster software, we searched for small delections-micro-deletion (20 bp or less) that are definitely pathogenic and dispersed in the large base deletion mutation of the allele from this proband's mother, and then used the online tool HGMD to find the corresponding known diseases.

\section{Results}

\section{hepatic pathology}

The patient's hepatic lobular acinar morphology was normal, with mild lymphocyte infiltration around the portal vein in the portal area, moderate fibrosis, and lack of interlobular bile ducts accompanied by arteries (Fig. 1a). There were also obvious widening and venous fibrosis around the portal vein, and slight bile duct hyperplasia at the edge of the portal area (Fig. 1 b, 1 e). Infiltration of inflammatory cells around the portal vein, mainly CD3+ (mature T lymphocytes) and CD20+ (B lymphocytes) cytes (Fig. $1 \mathrm{C}, \mathrm{d}$ ). The expression of hepatocyte ABCB4/MDR3 protein was basically normal, and there was no obvious deletion (Fig. $1 \mathrm{f}$ ).

\section{Target gene acquisition}

The test results were further screened, and the pathogenic site was found in the ABCB4 gene. One of the mutation information is c.2696C $>\mathrm{G}$ (p.Ala899Gly), which means, the 2696th base of the ABCB4 gene is mutated from $C$ to $G$, which encoded the 899th amino acid from Ala (alanine) to Gly (glycine); the location of the mutation site is on chromosome 7 (87043020), exon 22, which is a heterozygous mutation. The other is a large base deletion: wes [hg19]7q21.12 (87032513-87033422) $\times 1$. The deletion site involves an approximately 909 bp fragments of exon 27 on the long arm of chromosome 7 of the ABCB4 gene.

\section{Sanger sequencing verification results}

Forward sequencing of the target gene of $A B C B 4$ in the proband indicated that base 2696 was mutated from $C$ to $G$ (Fig. 2a) $\varangle$ Reverse sequencing showed that the base at this site was mutated from $G$ to $C$ (Fig. 2b). It was further verified that the base of the proband's father was mutated from $C$ to $G$ (Fig. 2c), and he was a carrier of the corresponding missense variant (Fig. 2c). The mother of the proband had normal 
bases at this site and was wild-type (Fig. 2d). It was confirmed that the missense variant c. $2696 \mathrm{C}>\mathrm{G}$ (p.Ala899Gly) of the patient came from his father. After searching the American PubMed and HGMD professional edition databases, it is confirmed that such mutations are new mutations that have not been reported.

\section{Semi-quantitative PCR verification results for specific gene deletion mutations}

we analysed the 5 gene segments transcription of ABCA4 gene exon 27 via Semi-quantitative PCR.The results showed that compared with normal control, the relative quotient (RQ) of exon $27(1,2)$ of the ABCB4 gene in the proband and her mother was both close to 0.5 which demonstrated the proband's heterozygous deletions originated from her mother. Simultaneously, the RQ of the exon 27 in her father was nearly 1 which proved that her father was normal (Fig. 3). Therefore, we concluded that the patient's large deletion mutation[ wes[hg19]7q21.12(87032513-87033422)×1] was inherited from her mother. After searching the American PubMed and HGMD professional edition databases, we confirm that such mutations are new mutations that have not been reported.

\section{Prediction of the MDR3 protein structure by SWISS-MODEL}

ABCB4 mainly encodes multidrug resistance protein 3 (MDR3), which is mainly expressed on the bile duct membrane, and is used as a phospholipid efflux pump. The protein structure prediction results suggested that, compared with wild type (Fig. 4a1, 4b1), one of the genes showed a missense mutation (c.

$2696 \mathrm{C}>\mathrm{G}$ ), the protein side chain produced was missing a methyl $(-\mathrm{CH} 3)$, and $\mathrm{CH} 3$ was replaced by $\mathrm{H}$ (Fig. $4 \mathrm{a} 2,4 \mathrm{~b} 2$ ). At the same time, deletion mutation (c.3487_3571del) was found in another gene which resulted in serious destruction of MDR3 protein- large alpha helix and small beta sheet deletions (Fig. 4 $c 1,4 c 2,4 d 1,4 d 2)$. Significant changes in the structure will affect the protein's transmembrane transport of phospholipids which causes the disease.

\section{Prediction of pathogenicity by Mutation Taster, PROVEAN and other online software}

After using relevant webpage software to predict the pathogenicity of the mutant genes, both alleles are pathogenic, and the pathogenicity of the deletion mutation is more significant, see Tab 4 . If the previously reported small-scale base deletion fragment (20bp or less) happens to fall in the base sequence of the patient's deletion mutation, use the HGMD and Mutation Taste software to find the corresponding serial number and reported Phenotype, see Tab 5. It is further predicted that the diseases that can cause PFIC, Low phospholipid associated cholelithiasis and intrahepatic cholestasis of pregnancy.

\section{Recent clinical follow-up}

After taking UDCA for 29 months, the patient 's latest B-ultrasound (September 14, 2021) displayed that cirrhosis was improved, the hardness of the liver decreased, the internal diameter of splenic vein returned to normal (Inner diameter of splenic vein: from $90 \mathrm{~mm}$ [February of 2019] to $40 \mathrm{~mm}$ [September of 2021]) and the spleen retracted(length of spleen under rib arch: from35mm[February of 2019] to 
$18 \mathrm{~mm}[$ September of 2021]). In the results of hepatic function (September 5, 2021) indicated that all except forbile acid were basically close to normal (Table 1 ).

\section{Discussion}

The ABCB4 gene is localized on chromosome 7q21 which is about $74 \mathrm{~kb}$ in length, contains 28 exons, and encodes the MDR3 protein[14]. Mutations of ABCB4 gene includes missense mutations, nonsense mutations, deletion mutations, insertion mutations and splice site mutations. Single allele mutations often occur in ABCB4 gene mutation-related diseases, including low phospholipid-associated cholelithiasis (LPAC), intrahepatic cholestasis of pregnancy (ICP), oral contraceptive-induced cholestasis (CIC), and PFIC3[15].

ABCB4 gene contains 28 exons and 27 coding sequences, it is also called MDR3 gene, which is a supergene family of $A B C$ transfer proteins, a member of the $P$ glycoprotein gene family [16-18]. AS an output pump for phospholipid, MDR3 encoded by the ABCB4 gene is mainly expressed on the cytomembrane of biliary duct, and responsible for transporting phospholipid from hepatocytes to duct capillary[19].Under normal circumstances, MDR3 transports phospholipid to bile, emulsifies bile salt and cholesterol, and forms particles together with bile salt to increase the hydrophilicity of bile salt, thereby reducing the descaling effects of bile salt and protecting cytomembrane of biliary duct. Preventing the toxic damage of bile salt plays an important role in protecting the liver [20]. ABCB4 gene mutations can cause the truncation, instability, misfoldin of the MDR3 [21] which leads to abnormal transport of phospholipid on different levels. The lack of phospholipids and phosphatidylcholine in bile results in bile salt released from bile ingredients, the increase in the ratio of cholesterol to phosphatidylcholine, easy formation of cholesterol crystals and obstruction of small bile duct [22]. Patients of PFRIC3 generally have cholestasis, ductular hyperplasia, inflammatory infiltration and portal fibrosis who finally progress to cirrhosis and portal hypertension, eventually die of hepatic failure [23].

The disease caused by ABCB4 gene abnormality is an autosomal recessive inherited disease. Generally, the disease occurs only when both alleles are abnormal. We confirmed the allelic heterozygous mutation in the $A B C B 4$ gene of this patient through exome capture and sequencing, one of which occurred in the 22nd exon of the ABCB4 gene, with a missense mutation c. $2696 \mathrm{C}>\mathrm{G}$; the other $A B C B 4$ Gene has a large deletion mutation, namely c.3487_3571del. It was verified by Sanger sequencing that the missense mutant came from the patient's father; it was confirmed by semi-quantitative PCR technology that the deletion mutation came from the patient's mother. Two mutants have not been reported in the literature. We further found through SWISS-MODEL software protein structure prediction that the missense mutant c. $2696 C>G$ resulted in a missing methyl group on the side chain of the MDR3 protein; gene deletion mutation c.3487_3571del resulted in the production of MDR3 protein alpha helix and beta sheet Structural damage, serious damage to protein structure. We speculated that the structurally abnormal MDR3 protein cannot bind to ATP and obtain energy through ATP decomposition, which affects the active transmembrane transport of phospholipids, resulting in a reduction in phospholipid content in bile and damage to biliary tract cells. 
We have not been able to confirm what is the pathogenic mechanism of her heterozygous mutations, and what are the abnormalities in the function and structure of her MDR3 protein?. Judging from the pathogenicity predicted by several online prediction software, this $A B C B 4$ gene heterozygous mutations have significant pathogenicity. HGMD and Mutation Taste online software predicts that the patient's heterozygous mutations can lead to three diseases, PFRIC3, cholestasis of pregnancy, and lowphospholipid-related cholelithiasis. The clinical feature of this patient is that the appearance of cholestatic cirrhosis in youth (compensation period, excluding other factors), her mother and her mother's family had a short history of cholestasis during pregnancy (specifically unknown).

After UDCA treatment for more than 7 months, there were still lack of interlobular bile ducts in the portal area, and slight hyperplasia of bile capillary, infiltration of chronic inflammatory cells in the portal area with peripheral fibrosis in her hepatic biopsy tissues, which were consistent with previous reports of PFRIC3 pathology [22]. MDR3 can be expressed without loss in PFRIC3 patients. In this case, the MDR3 expression was basically normal, so it was still uncertain whether it was the efficacy of treatment or MDR3 itself was not affected. Indeed, performing first hepatic pathological biopsy before treatment should be more convincing. Based on above evidence, This patient was considered to be more in line with PFRIC3 caused by mutations of ABCB4 gene.

UDCA, rifampicin, phenobarbital and other drugs are mainly used for PFIC-3 in clinical treat which can delay the progression of cirrhosis. Among them, UDCA has the greatest application value. On one hand, non-detergent and hydrophilic UDCA is secreted into bile, partially replacing the primary bile salt of hydrophobic and detersive bile (cholic acid and chenodeoxycholic acid);on the other hand, UDCA shows the effect to enhance the expression of MDR3 on cytomembrane of the biliary ducts[24]. Some studies also have found that UDCA cannot increase the mRNA level of ABCB4 gene, but can increase its hepatic protein expression level [25]. Patients who respond to UDCA mainly have the mild genetic defects (for example, the ABCB4 missense mutation leads to residual MRD3 function) and simultaneously have the residual phospholipid concentration in bile (threshold of $7 \%$ of total biliary lipids)[26]..UDCA can promote the excretion of bile, increase the phospholipid concentration of bile,] dissolve cholesterol crystals and protect cell membranes from bile salt toxicity [22].Compared with other drugs, UDCA is less toxic, more hydrophilic, and has less damage to biliary tract cell. With definite therapeutic effect, long-term use of UDCA can delay the progression of cirrhosis, delay the time for hepatic transplantation [17, 27].However, patients with some types of mutations (nonsense mutations, deletion mutations, etc., which cause all defects in MRD3 expression) are more seriously ill, and UDCA replacement therapy is not enough to reduce the increased bile salt toxicity because of their phospholipid-free bile[23, 28]. For this case, after 7 months treatment of UDCA, her hepatic pathology had shown there were no obvious pseudolobules and regenerative nodules which implied that her cirrhosis may be better than before. Twenty-nine months treatment of UDCA caused her hepatic function nearly recovered, ultrasound indicated cirrhosis significantly improved. Due to patient's nonacceptance, we had no further hepatic pathology to verify the clinical effects. But indeed UDCA was effective for this case. On the one hand, it is considered that the patient has a heterozygous mutation, although one of the chromosomes is a deletion mutation, but the other is a missense mutation; on the other hand, the patient underwent hepatic pathological examination 
after UDCA treatment, MDR3 expression was close to normal. We speculated that structure and function of MDR3 was relatively less impaired [7]. UDCA indeed enhances this patient's capability of MDR3.

For patient who is young and not in a bad way at the time of onset with history of familial cholestasis, whose clinical features were repeated cholestasis, especially increased GGT and bile acid, he or she should be highly suspected of suffering from PFIC3. Although there are many diseases caused by ABCB4 gene abnormalities, their manifestations may be relatively insidious. If PFIC3 can be diagnosed early and accurately, the effective therapeutic intervention (UDCA) may significantly improve the prognosis and delay the occurrence of end-stage hepatic disease. Of course, we still need more clinical data and animal experiments to confirm the relationship, mechanism and clinical characteristics between different variants of the ABCB4 gene and different types of diseases in order to better discover and diagnose such diseases.

\section{Abbreviations}

ABC: ATP-binding cassette; ABCB4: ATP binding cassette subfamily B member 4 gene; Albumin: A;Ala: alanine; ALT: alanine aminotransferase; ALP: alkaline phosphatase; AST: aspartate aminotransferase; BSEP: bile salt exporter pump; CIC: contraceptive-induced cholestasis; D.Bil: Direct bilirubin; Globulin: G; Gly: glycine; GGT: gamma glutamyl transpeptisae; I.Bil: Indirect bilirubin; ICP: intrahepatic cholestasis of pregnancy; LDH: Lactate dehydrogenase; LPAC: low phospholipid-associated cholelithiasis; MDR3: multidrug resistance class III; NGS: The Next Generation Sequencing; PFIC: Progressive familial intrahepatic cholestasis; TBA: Total bile acid; T.Bil: total bilirubin; TP: total protein; UDCA: ursodeoxycholic acid

\section{Declarations}

\section{Acknowledgements}

Not applicable.

\section{Authors' contributions}

Fei Qiao reviewed the case and wrote the manuscript. Weiting Lu drafted the tables and figures; Feng Ren and Haoran Yang contributed to the acquisition of literature and biological information analysis. Guiling Mo performed the genetic testing. Shuangshuang Wang performed pathological examination and analyzed pathological results. Lina Liu provided the clinical data, supervised this research and edited the manuscript. Xiangtao Xu carried out the pathological biopsy operation and reviewed the pathology. The authors read and approved the final version of the manuscript.

\section{Funding}

None. 
Availability of data and materials

The data that support the findings of this study are available from the corresponding author (Lina Liu and Xiangtao $\mathrm{Xu}$ ) upon reasonable request.

\section{Ethics approval and consent to participate}

All study procedures were in accordance with the ethical standards of the responsible committee on human experimentation (institutional and national) and with the guidelines and regulations of the Helsinki and Human Research Protection Program.

\section{Consent for publication}

The consent for publication has been obtained from the institutional board from the Affiliated Hospital of Nanjing University of Chinese Medicine. All authors agree to publish.

\section{Competing interests}

The authors do not have any conflicts of interest to declare.

\section{Author details}

${ }^{1}$ Department of Hepatology, The Affiliated Hospital of Nanjing University of Chinese Medicine, Nanjing, China. ${ }^{2}$ First Clinical Medical College, Nanjing University of Chinese Medicine, Nanjing, China. ${ }^{3}$ Medical Laboratory Science, Guangzhou KingMed Center For Clinical Laboratory Co.,Ltd, Guangzhou, China. ${ }^{4}$ Department of Pathology, The Affiliated Hospital of Nanjing University of Chinese Medicine, Nanjing, China.

\section{ORCID}

Fei Qiao https://orcid.org/0000-0003-0204-0180

Weiting Lu https://orcid.org/0000-0002-1149-7003

Feng Ren https://orcid.org/0000-0002-0136-9542

Haoran Yang https://orcid.org/0000-0001-8040-6518

Guiling Mo https://orcid.org/0000-0003-0220-3162

Shuangshuang Wang https://orcid.org/0000-0001-9815-8233

Lina Liu https://orcid.org/0000-0002-5813-1375

Xiangtao Xu https://orcid.org/0000-0003-0575-1578 


\section{References}

1. Davit-Spraul A, Gonzales E, Baussan C, Jacquemin E. Progressive familial intrahepatic cholestasis. Orphanet J Rare Dis. 2009;4:1.

2. van Mil SW, Klomp LW, Bull LN, Houwen RH. FIC1 disease: a spectrum of intrahepatic cholestatic disorders. Semin Liver Dis. 2001;21(4):535-44.

3. Bourke B, Goggin N, Walsh D, Kennedy S, Setchell KD, Drumm B. Byler-like familial cholestasis in an extended kindred. Arch Dis Child. 1996;75(3):223-7.

4. Strautnieks SS, Kagalwalla AF, Tanner MS, Knisely AS, Bull L, Freimer N, et al. Identification of a locus for progressive familial intrahepatic cholestasis PFIC2 on chromosome 2q24. Am J Hum Genet. 1997;61(3):630-3.

5. Li Q, Sun Y, van ISCD. A Link between Intrahepatic Cholestasis and Genetic Variations in Intracellular Trafficking Regulators. Biology (Basel). 2021;10(2).

6. Sundaram SS, Sokol RJ. The Multiple Facets of ABCB4 (MDR3) Deficiency. Curr Treat Options Gastroenterol. 2007;10(6):495-503.

7. Delaunay JL, Durand-Schneider AM, Dossier C, Falguieres T, Gautherot J, Davit-Spraul A, et al. A functional classification of $A B C B 4$ variations causing progressive familial intrahepatic cholestasis type 3. Hepatology. 2016;63(5):1620-31.

8. Jansen PL, Muller M. Genetic cholestasis: lessons from the molecular physiology of bile formation. Can J Gastroenterol. 2000;14(3):233-8.

9. Jankowska I, Socha P. Progressive familial intrahepatic cholestasis and inborn errors of bile acid synthesis. Clin Res Hepatol Gastroenterol. 2012;36(3):271-4.

10. Morotti RA, Suchy FJ, Magid MS. Progressive familial intrahepatic cholestasis (PFIC) type 1, 2, and 3: a review of the liver pathology findings. Semin Liver Dis. 2011;31(1):3-10.

11. Jacquemin E. Progressive familial intrahepatic cholestasis. Clin Res Hepatol Gastroenterol. 2012;36 Suppl 1:S26-35.

12. Santana A, Waiswo M. The genetic and molecular basis of congenital cataract. Arq Bras Oftalmol. 2011;74(2):136-42.

13. Richards S, Aziz N, Bale S, Bick D, Das S, Gastier-Foster J, et al. Standards and guidelines for the interpretation of sequence variants: a joint consensus recommendation of the American College of Medical Genetics and Genomics and the Association for Molecular Pathology. Genet Med. 2015;17(5):405-24.

14. Trauner M, Fickert $P$, Wagner M. MDR3 (ABCB4) defects: a paradigm for the genetics of adult cholestatic syndromes. Semin Liver Dis. 2007;27(1):77-98.

15. Pasmant E, Goussard P, Baranes L, Laurendeau I, Quentin S, Ponsot P, et al. First description of ABCB4 gene deletions in familial low phospholipid-associated cholelithiasis and oral contraceptivesinduced cholestasis. Eur J Hum Genet. 2012;20(3):277-82. 
16. Sun HZ, Shi H, Zhang SC, Shen XZ. Novel mutation in a Chinese patient with progressive familial intrahepatic cholestasis type 3. World J Gastroenterol. 2015;21(2):699-703.

17. Wang Z, Yang F, Wang J, Ma X. A Novel Mutation of ABCB4 in Progressive Familial Intrahepatic Cholestasis 3: Like Mother, Like Daughter. J Clin Gastroenterol. 2016;50(4):353-4.

18. Gordo-Gilart R, Andueza S, Hierro L, Martinez-Fernandez P, D'Agostino D, Jara P, et al. Functional analysis of ABCB4 mutations relates clinical outcomes of progressive familial intrahepatic cholestasis type 3 to the degree of MDR3 floppase activity. Gut. 2015;64(1):147-55.

19. Kim TH, Park HJ, Choi JH. Functional Characterization of ABCB4 Mutations Found in Low Phospholipid-Associated Cholelithiasis (LPAC). Korean J Physiol Pharmacol. 2013;17(6):525-30.

20. Groen A, Romero MR, Kunne C, Hoosdally SJ, Dixon PH, Wooding C, et al. Complementary functions of the flippase ATP8B1 and the floppase ABCB4 in maintaining canalicular membrane integrity. Gastroenterology. 2011;141(5):1927-37 e1-4.

21. Saleem K, Cui Q, Zaib T, Zhu S, Qin Q, Wang Y, et al. Evaluation of a Novel Missense Mutation in ABCB4 Gene Causing Progressive Familial Intrahepatic Cholestasis Type 3. Dis Markers. 2020;2020:6292818.

22. Davit-Spraul A, Gonzales E, Baussan C, Jacquemin E. The spectrum of liver diseases related to ABCB4 gene mutations: pathophysiology and clinical aspects. Semin Liver Dis. 2010;30(2):134-46.

23. Jacquemin E, De Vree JM, Cresteil D, Sokal EM, Sturm E, Dumont M, et al. The wide spectrum of multidrug resistance 3 deficiency: from neonatal cholestasis to cirrhosis of adulthood. Gastroenterology. 2001;120(6):1448-58.

24. Dilger K, Denk A, Heeg MH, Beuers U. No relevant effect of ursodeoxycholic acid on cytochrome P450 3A metabolism in primary biliary cirrhosis. Hepatology. 2005;41(3):595-602.

25. Oude Elferink RP, Paulusma CC. Function and pathophysiological importance of ABCB4 (MDR3 Pglycoprotein). Pflugers Arch. 2007;453(5):601-10.

26. de Vree JM, Jacquemin E, Sturm E, Cresteil D, Bosma PJ, Aten J, et al. Mutations in the MDR3 gene cause progressive familial intrahepatic cholestasis. Proc Natl Acad Sci U S A. 1998;95(1):282-7.

27. Lincke CR, Smit JJ, van der Velde-Koerts T, Borst P. Structure of the human MDR3 gene and physical mapping of the human MDR locus. J Biol Chem. 1991;266(8):5303-10.

28. Van Nieuwkerk CM, Elferink RP, Groen AK, Ottenhoff R, Tytgat GN, Dingemans KP, et al. Effects of Ursodeoxycholate and cholate feeding on liver disease in FVB mice with a disrupted mdr2 Pglycoprotein gene. Gastroenterology. 1996;111(1):165-71.

\section{Tables}

Table 1 Trends in liver function 


\begin{tabular}{|c|c|c|c|c|c|c|c|c|}
\hline $\begin{array}{l}\text { Liver } \\
\text { function }\end{array}$ & $\begin{array}{l}\text { October } \\
4,2018\end{array}$ & $\begin{array}{l}\text { January } \\
8,2019\end{array}$ & $\begin{array}{l}\text { March } \\
23 \\
2019\end{array}$ & $\begin{array}{l}\text { May } \\
2, \\
2019\end{array}$ & $\begin{array}{l}\text { August } \\
6,2019\end{array}$ & $\begin{array}{l}\text { June } \\
3, \\
2020\end{array}$ & $\begin{array}{l}\text { November } \\
6,2020\end{array}$ & $\begin{array}{l}\text { September } \\
5,2021\end{array}$ \\
\hline $\begin{array}{l}\text { ALT } \\
(\mathrm{U} / \mathrm{L})\end{array}$ & 19 & $110 \mathrm{H}$ & $33 \mathrm{H}$ & $42 \mathrm{H}$ & 31 & 25 & 22 & 24 \\
\hline $\begin{array}{l}\text { AST } \\
(\mathrm{U} / \mathrm{L})\end{array}$ & $57 \mathrm{H}$ & $93 \mathrm{H}$ & $42 \mathrm{H}$ & $55 \mathrm{H}$ & $34 \mathrm{H}$ & 26 & 25 & 21 \\
\hline $\mathrm{TP}(\mathrm{g} / \mathrm{L})$ & 75.6 & $74.9 \mathrm{H}$ & 75.04 & 77.34 & 68.58 & 69.92 & 65.78 & 67.41 \\
\hline$A(g / L)$ & 39.6 & 42.5 & 39.2 & 39 & 39.9 & 40.07 & 41.8 & 40.7 \\
\hline $\mathrm{G}(\mathrm{g} / \mathrm{L})$ & $36.0 \mathrm{H}$ & $32.4 \mathrm{H}$ & $35.84 \mathrm{H}$ & $\begin{array}{l}38.34 \\
\mathrm{H}\end{array}$ & 28.68 & 29.85 & 23.98 & 26.7 \\
\hline $\begin{array}{l}\mathrm{LDH} \\
(\mathrm{g} / \mathrm{L})\end{array}$ & 180 & 208 & $214 \mathrm{H}$ & $236 \mathrm{H}$ & 220 & 170 & 163 & 212 \\
\hline $\begin{array}{l}\text { ALP } \\
\text { (U/L) }\end{array}$ & $136 \mathrm{H}$ & $167 \mathrm{H}$ & 133 & 112 & 103 & 75 & 74 & 68 \\
\hline $\begin{array}{l}\text { GGT } \\
(\mathrm{U} / \mathrm{L})\end{array}$ & $197 \mathrm{H}$ & $301 \mathrm{H}$ & $157 \mathrm{H}$ & $55 \mathrm{H}$ & $41 \mathrm{H}$ & $46 \mathrm{H}$ & $38 \mathrm{H}$ & 31 \\
\hline $\begin{array}{l}\text { TBA } \\
(\mathrm{U} / \mathrm{L})\end{array}$ & 11.9 & 8.5 & $75.4 \mathrm{H}$ & $\begin{array}{l}158.4 \\
H\end{array}$ & $86.1 \mathrm{H}$ & $\begin{array}{l}15.5 \\
\mathrm{H}\end{array}$ & $25.8 \mathrm{H}$ & $15 \mathrm{H}$ \\
\hline $\begin{array}{l}\text { T.Bil } \\
(\mu \mathrm{mol} / \mathrm{L})\end{array}$ & 22.7 & 11.1 & $22.62 \mathrm{H}$ & $\begin{array}{l}51.61 \\
\mathrm{H}\end{array}$ & 14.94 & 21.01 & 14.79 & 20.72 \\
\hline $\begin{array}{l}\text { D.Bil } \\
(\mu \mathrm{mol} / \mathrm{L})\end{array}$ & 12.7 & 4.8 & 7.55 & $\begin{array}{l}34.76 \\
\mathrm{H}\end{array}$ & 3.47 & 3.24 & 3.05 & 5.44 \\
\hline $\begin{array}{l}\text { I.Bil } \\
(\mu \mathrm{mol} / \mathrm{L})\end{array}$ & 10.02 & 6.3 & 15.07 & 18.65 & 11.47 & 17.77 & 11.74 & 15.28 \\
\hline
\end{tabular}

Abbreviations: ALT, alanine aminotransferase; AST, aspartate aminotransferase; TP, total protein; A, Albumin; G, Globulin; LDH,Lactate dehydrogenase; ALP, alkaline phosphatase; GGT, gamma glutamyl transpeptisae; TBA, Total bile acid; T.Bil, total bilirubin; D.Bil, Direct bilirubin; I.Bil, Indirect bilirubin.

Table 2 PCR conditions Amplification and sequencing analysis primers and

\begin{tabular}{|c|c|c|c|c|}
\hline Exon & Primer sequence $₫ 5$ '-3 ') & Annealing temperature $\rrbracket^{\circ} \mathrm{C} \rrbracket$ & Polymerase & product $\triangle \mathrm{bp} \mathbb{Z}$ \\
\hline \multirow[t]{2}{*}{22} & TGACAGAGCCCTAGGGCTTA & \multirow[t]{2}{*}{60} & \multirow[t]{2}{*}{ AmpliTag } & \multirow[t]{2}{*}{$279 b p$} \\
\hline & CATTGTTTGGAGCAGCAGAG & & & \\
\hline
\end{tabular}


Table 3 PCR primer sequence

\begin{tabular}{|lll|}
\hline Gene & Primer sequence & PCR product length \\
\hline ABCB4.Ex27.1 & F-GCATAAGCCCTGCAAACTG & $91 \mathrm{bp}$ \\
& R-GGGGAGCTAGATTTTGAGAGAG & \\
ABCB4.Ex27.2 & F-TTCTGATCTTTCATAGTGTGCTGTGTA & 88bp \\
& R-TGTGGTATACGCAGTCTGTCATTG & \\
ABCB4.Ex27.3 & F-CAAAAGAAAAAAGCTGCACAAATTAC & $89 \mathrm{bp}$ \\
& R-TCTTCTGTTTAACTAGTTCCCTTTTGAGT & \\
ABCB4.Ex27.4 & F-AGACAACCTCAAATCCTCCTG & $74 \mathrm{bp}$ \\
& R-GGCCACACACACCTTTTCA & \\
\hline ABCB4.Ex27.5 & F-CCAGGAGTAGAATTGTGGAGCAG & $83 \mathrm{bp}$ \\
& R-GGATGGCCACTCTGGAAAAC & \\
\hline
\end{tabular}

Table 4 Pathogenicity predictions for mutation by computational algorithms

\begin{tabular}{|lll|}
\hline $\begin{array}{l}\text { Computational } \\
\text { algorithms }\end{array}$ & ABCB4:c.2696C>G(p.Ala899Gly) & $\begin{array}{l}\text { ABCB4:wes[hg19]7q21.12(87032513- } \\
\mathbf{8 7 0 3 3 4 2 2 ) \times 1}\end{array}$ \\
\cline { 2 - 3 } & Prediction (Score) & Prediction (Score) \\
\hline PROVEAN & Deleterious(-3.633) & Deleterious(-394.685) \\
\hline Mutation Taster & Disease causing & Long InDel \\
\hline
\end{tabular}

Table 5 Phenotype predictions for wes [hg19]7q21.12 (87032513-87033422) ×1 by HGMD and Mutation Taster 


\begin{tabular}{|ll|}
\hline Accession Number & Phenotype \\
\hline CD105816 & Low phospholipid associated cholelithiasis \\
\hline CM075959 & Intrahepatic cholestasis, familial progressive \\
\hline CM105805 & Intrahepatic cholestasis of pregnancy \\
\hline CM105807 & Low phospholipid associated cholelithiasis \\
\hline CM119907 & Intrahepatic cholestasis, familial progressive \\
\hline
\end{tabular}

\section{Figures}

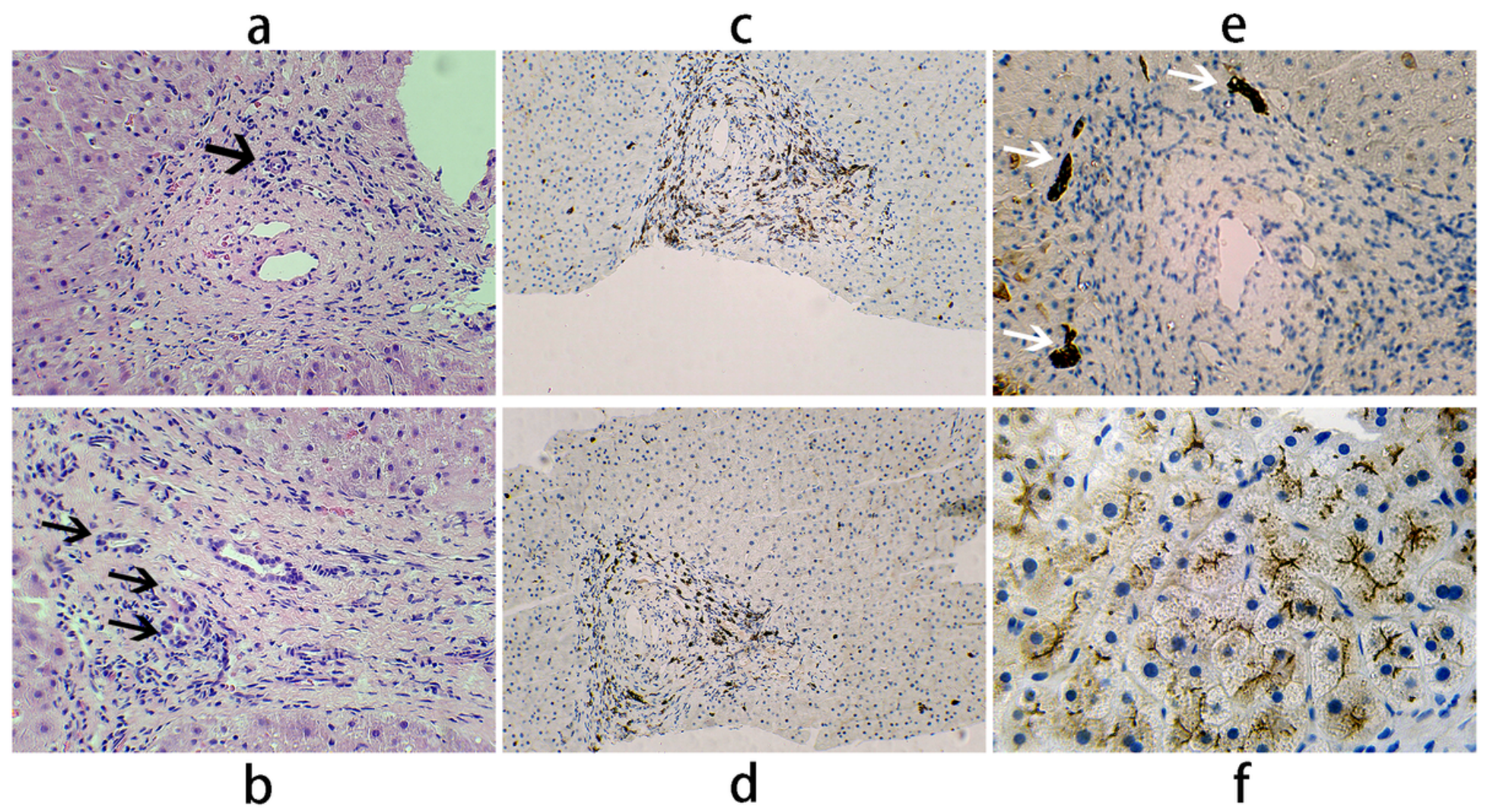

\section{Figure 1}

hepatic pathology: (a)Unaccompanied interlobular bile ducts adjacent to arteries(Black arrow, 200x, Hematoxylin and Eosin stain); (b)Obviously widening and fibrosis around the portal vein, accompanied by bile duct hyperplasia(Black arrow, 200x, Hematoxylin and Eosin stain); (c, d) Inflammatory cell infiltration around portal vein: immunostaining of lymphocyte subtype CD3+ cells around portal vein(100x区 c);Immunostaining of lymphocyte subtype CD20+ cells around portal vein(100x囚d); (e) CK7 
immunohistochemical staining showed the lack of interlobular bile ducts in the portal area, and slight hyperplasia of bile capillary at the edge of the portal area(White arrow, 100x); (f) Immunohistochemical staining showed positive expression of MDR3 protein beside hepatocytes (500x)

\section{c.2696C>G(p.Ala899Gly)}

\section{a}

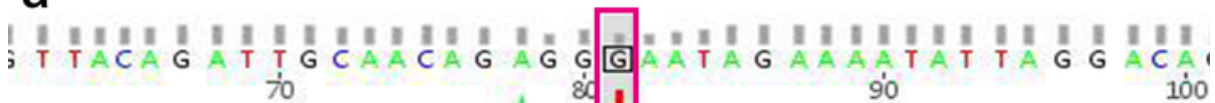

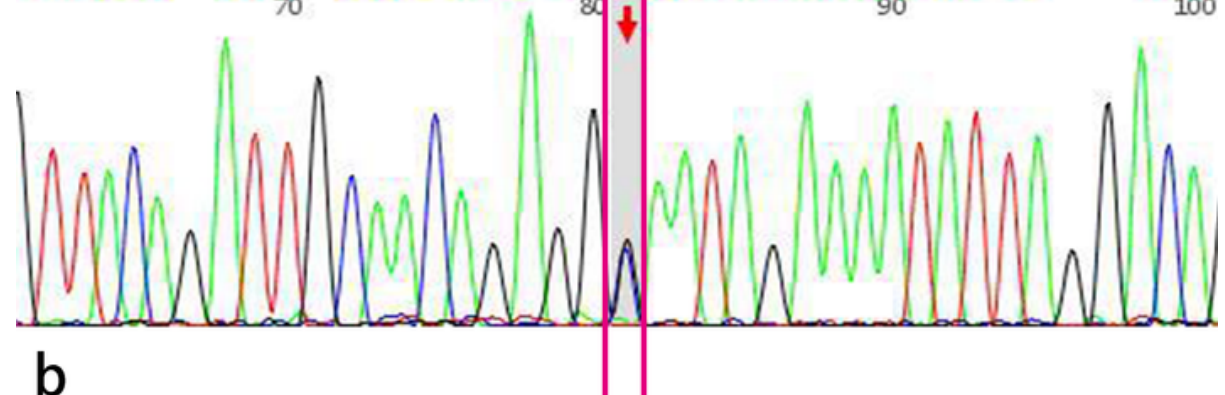

b

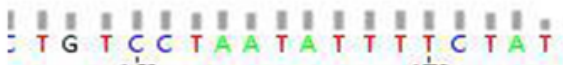

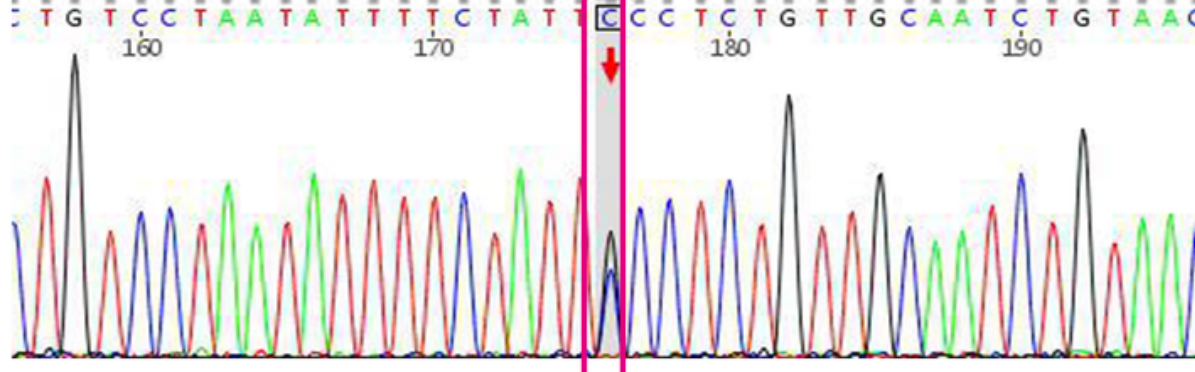

C

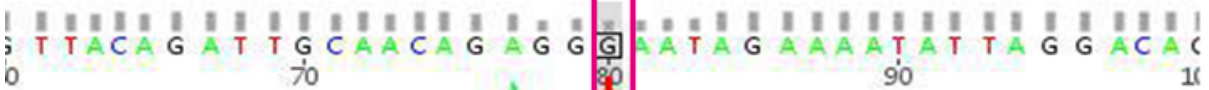

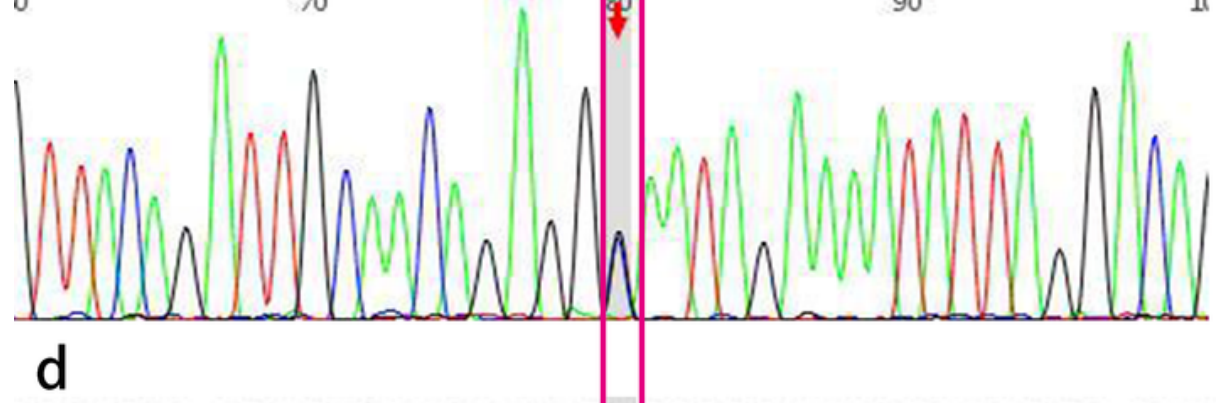

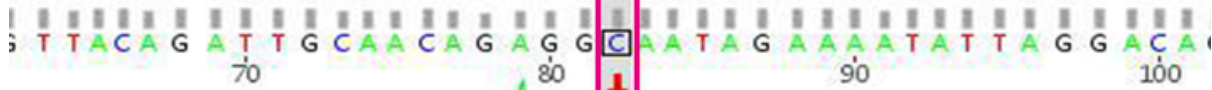

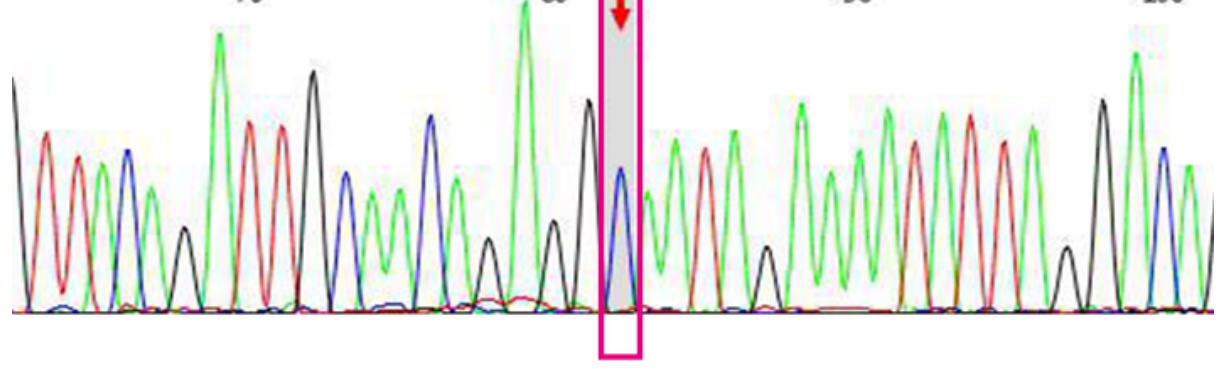

Figure 2

Sanger sequencing confirmed the variant of c.2696C>G (p.Ala899Gly) in ABCB4 
in proband's father but not in her mother: (a) Forward sequencing of the proband; (b)Reverse sequencing of the proband; (c) Sanger sequencing in the proband father showed this missense variant altered a Alanine (Ala ) residue at position 899 in the ABCB4 to a Glycine (Gly). (d)Sanger sequencing in the proband mother showed this site was wild type.

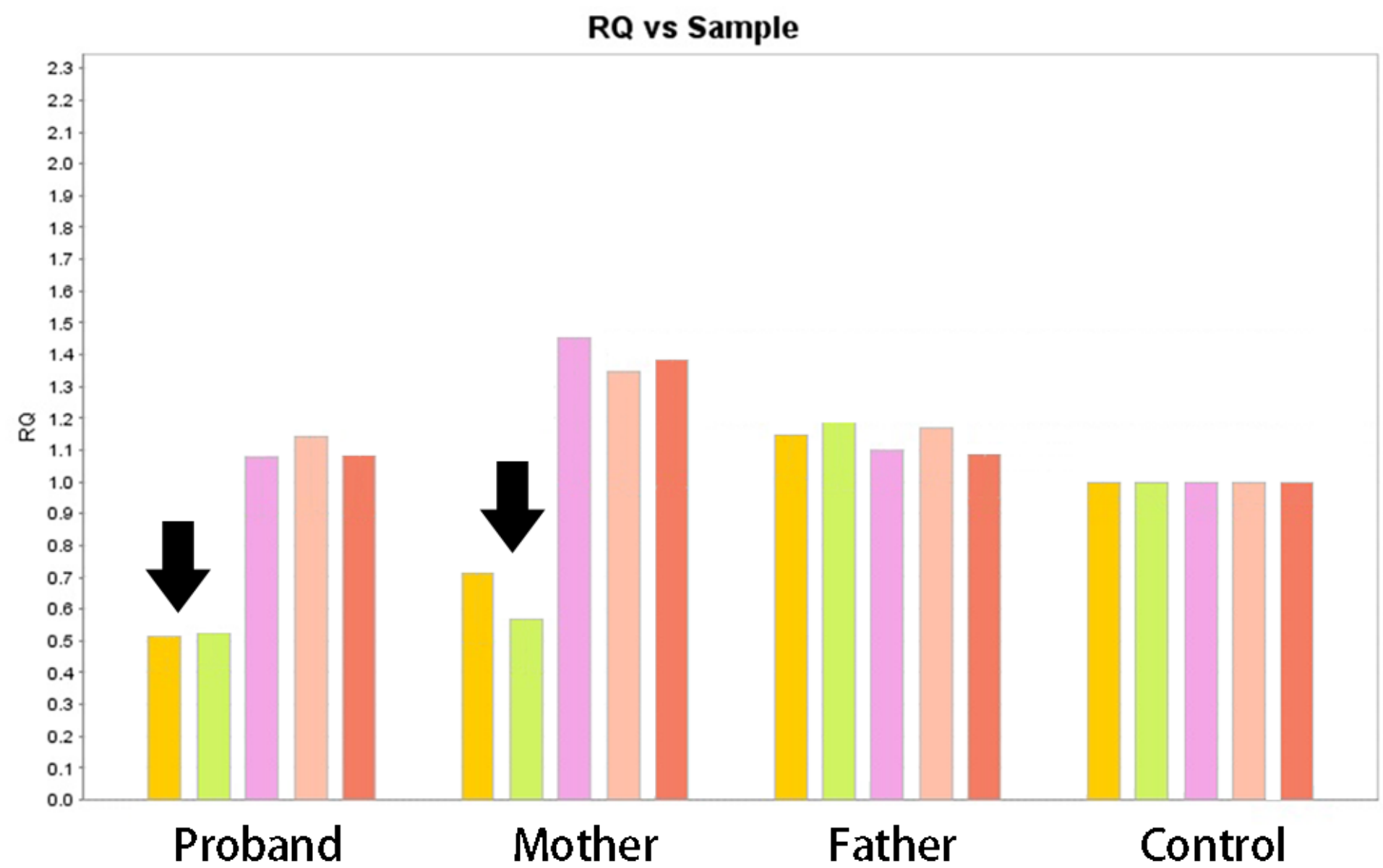

\begin{tabular}{l|l|l|l|l|l} 
ABCB4.Ex27.1 & ABCB4.Ex27.2 & ABCB4.Ex27.3 & ABCB4.Ex27.4 & ABCB4.Ex27.5
\end{tabular}

Figure 3

Real-time PCR of genes of interest. (RQ: relative quotient as determined by $\Delta \Delta$ Ct method) 
a1

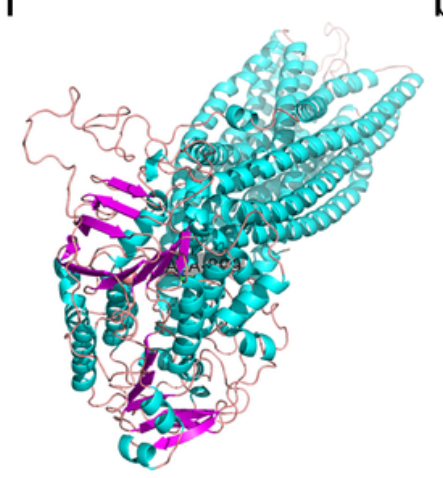

b1
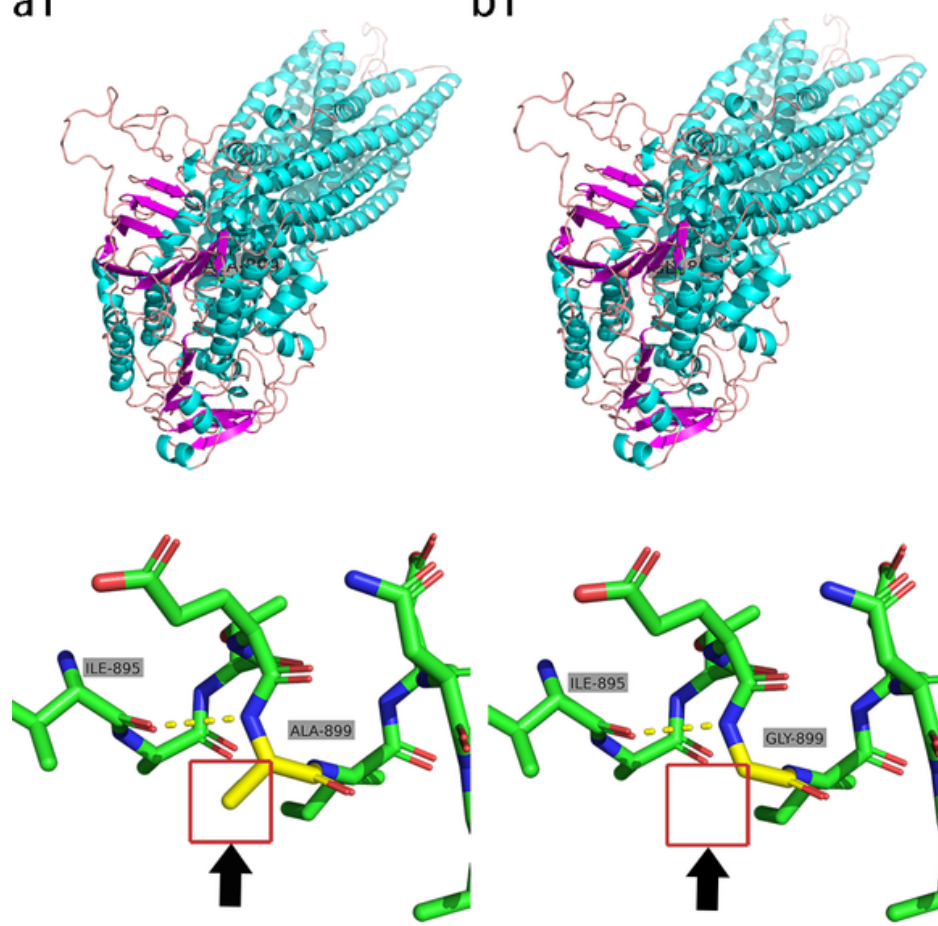

a2
C1

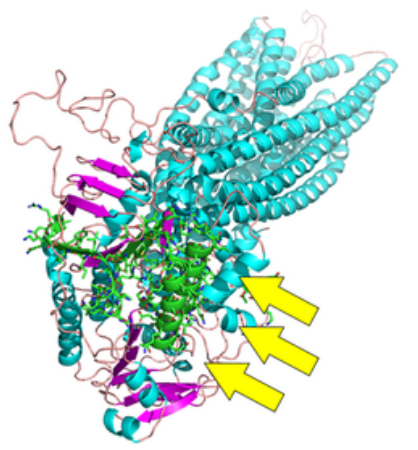

d1
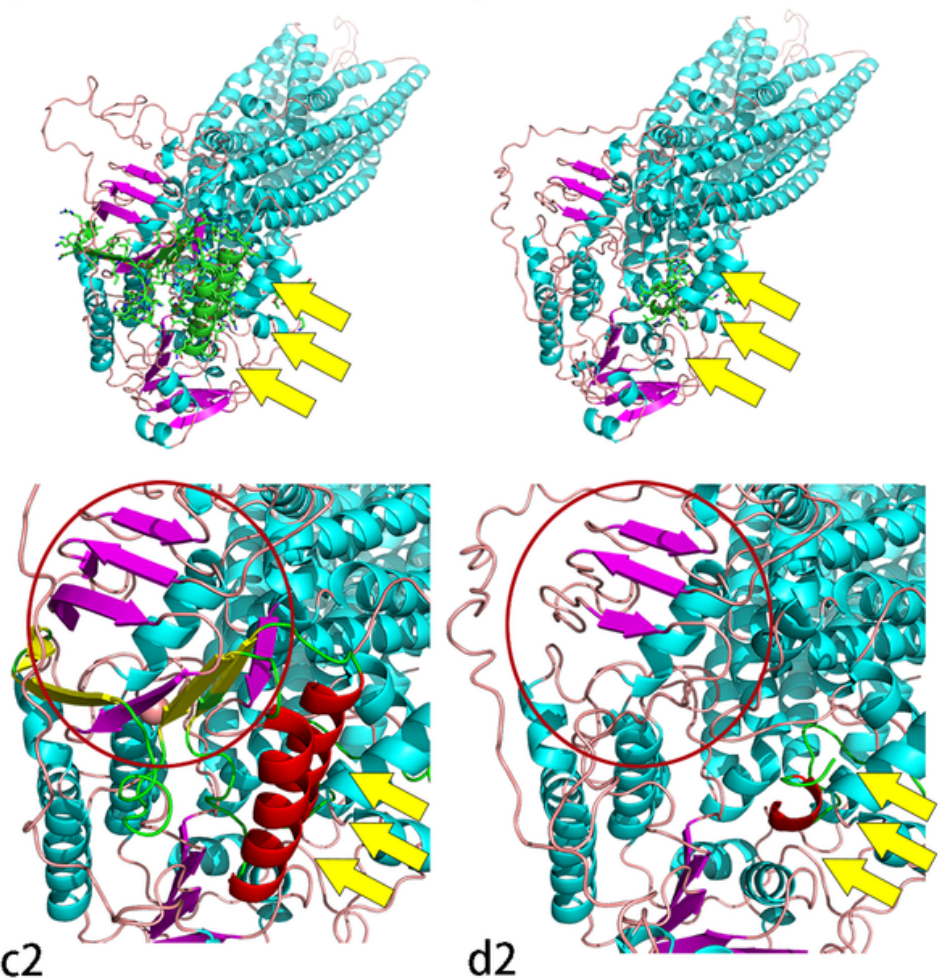

Figure 4

The whole three-dimensional predicted structure of MDR3 protein $(a 1, b 1, c 1, d 1)$ and local predicted structure $(a 2, b 2, c 2, d 2)$ 